\title{
Tomada de Decisão de Psicólogos em Situações de Suspeita de Abuso Sexual
}

\author{
Cátula Pelisoli ${ }^{1}$ \\ Tribunal de Justiça do Estado do Rio Grande do Sul, Passo Fundo, RS, Brasil \\ Débora Dalbosco Dell'Aglio \\ Departamento de Psicologia do Desenvolvimento e da Personalidade da Universidade Fede- \\ ral do Rio Grande do Sul, Porto Alegre, RS, Brasil
}

\begin{abstract}
Resumo
A tomada de decisão (TD) em situações de suspeita abuso sexual é uma tarefa complexa que envolve diferentes fontes de informação bem como influências subjetivas e não evidentes. A decisão tomada pelo profissional pode produzir consequências relevantes para as pessoas envolvidas. Um survey online com 95 psicólogos foi conduzido e avaliou a TD em um caso fictício de suspeita de abuso sexual e suas relações com variáveis cognitivas, sociodemográficas e profissionais. Além disso, a influência de características representativas de casos de abuso sexual na TD pelos profissionais foi avaliada a partir da Escala de Tomada de Decisão em Abuso Sexual Infantil - ETD ASI, desenvolvida para este estudo. Os resultados indicaram que os profissionais têm diferentes opiniões sobre o caso, ainda que expostos às mesmas informações. Idade, gênero, tempo de formação e tempo de prática não apresentaram correlação com a tomada de decisão. Entretanto, características representativas de abuso sexual tiveram relações significativas com a probabilidade avaliada do caso. Este resultado significa que a probabilidade de considerar o abuso sexual como verdadeiro é mais alta quanto mais características representativas possui o caso. A TD nessas situações, apesar de ser conduzida por profissionais todos os dias, é ainda desconhecida e merece futuras investigações.
\end{abstract}

Palavras-chave: Abuso sexual contra crianças e adolescentes, tomada de decisão, Psicologia Forense, Avaliação Psicológica.

\section{Decision-Making of Psychologists in Suspected Sexual Abuse Situations}

\begin{abstract}
Decision making in sexual abuse situation is a complex task involving different sources of information as well as non-obvious or subjective influences. The decision made by a professional can produce relevant consequences for the people involved. An online survey with 95 Brazilian bachelors in Psychology was conducted and it evaluated the decision-making in a fictitious case of sexual abuse and its relations with cognitive, sociodemographic and professional variables. In addition, the influence of the presence of representative characteristics of cases of sexual abuse in decision-making by professionals was evaluated from the Decision Making Scale in Child Sexual Abuse, an instrument developed for this study. The results indicated that the professionals have different opinions about the case, even if exposed
\end{abstract}

Endereço para correspondência: Rua Ramiro Barcelos, 2600/ sala 115, Santana, Porto Alegre, RS, Brasil 90035-003.E-mail: catulapelisoli@yahoo.com.bre dddellaglio@gmail.com

Financiamento: Conselho Nacional de Desenvolvimento Científico e Tecnológico (CNPq). 
to the same information. Age, gender, time of graduation and time of practice did not correlate with the decision made about the case. However, representative characteristics of sexual abuse had significant relations with the evaluated probability of the case. This result means that the probability of the case being considered true is higher when more representative characteristics has the case. Decision-making in these situations, despite being carried out by professionals every day, is still unknown and deserves further investigations.

Keywords: Sexual abuse, decision-making, Forensic Psychology, Psychological Evaluation.

\section{La Toma de Decisiones por los Psicólogos en Situaciones de Abuso Sexual}

\section{Resumen}

El proceso de toma de decisiones (TD) frente a situaciones de abuso sexual es una tarea compleja que envuelve diferentes fuentes de información así como influencias subjetivas y no evidentes. La decisión tomada por el profesional puede producir consecuencias relevantes para las personas involucradas. Un survey online con 95 psicólogos fue conducido y evaluó TD en un caso ficticio de abuso sexual y sus relaciones con variables cognitivas, sociodemográficas y profesionales. La influencia de la presencia de características representativas de casos de abuso sexual en la TD fue evaluada a partir de la Escala de Toma de Decisión en Abuso Sexual Infantil - ETD ASI, desarrollada para este estudio. Los resultados indicaron que los profesionales tienen diferentes opiniones sobre el caso, aunque expuestos a las mismas informaciones. Edad, género, tiempo de formación y tiempo de práctica no presentaron correlación con la TD. Sin embargo, características representativas de abuso sexual tuvieron relaciones significativas con la probabilidad evaluada del caso. Este resultado significa que la probabilidad de considerar el abuso como verdadero es más alta mientras más características representativas el caso tiene. La TD en esas situaciones, a pesar de conducida por profesionales todos los días, es aún desconocida y merece futuras investigaciones.

Palabras clave: Abuso sexual, Toma de decisiones, Psicología Forense, Evaluación Psicológica.

Alguns casos de abuso sexual infantil (ASI) apresentam informações claras e parecem não deixar dúvidas sobre a ocorrência do crime, como quando uma criança conta detalhadamente sobre o contato sexual com o adulto e/ou quando este adulto faz uma confissão (Berliner \& Conte, 1993). Entretanto, grande parte dos casos apresenta dados que levam os profissionais a se questionarem sobre se o abuso de fato ocorreu, se é uma falsa acusação, produto de uma falsa memória ou ainda se é uma suspeita infundada. Um exemplo de caso que gera dúvida é quando as crianças não têm habilidades suficientes para contar o que lhes aconteceu (linguística ou cognitiva, por exemplo) ou quando descrevem comportamentos que ocorreram, mas que podem não ter uma intenção sexualmente abusiva (como um banho ou outra atividade de higiene; Berliner \& Conte, 1993).
Outra possibilidade de uma situação de suspeita de ASI não ser verdadeira é pela existência de falsas acusações de abuso, disparadas por desentendimentos que suscitam sentimentos de vingança e raiva (Bernet, 2010). Falsas acusações também podem ser decorrentes de falsas memórias, espontaneamente criadas ou induzidas (Stein, 2010). Uma falsa memória pode ser espontânea se, por exemplo, sem influência de terceiros, há algum erro na fonte ou origem da informação, como quando uma pessoa se lembra de ter visto uma notícia na televisão, mas, na verdade, leu no jornal. As falsas memórias podem ser também induzidas, como em situações de alienação parental (que também é motivada por sentimentos de vingança e raiva), entrevistas sugestivas ou procedimentos psicoterapêuticos questionáveis (Loftus, 1997). Segundo Amendola (2009), as falsas acusações de abuso sexual 
em crianças envolvem necessariamente a sugestão de terceiros, caracterizando uma crença imposta pelo adulto de que a criança sofreu abuso, quando na realidade não sofreu. Para esta autora, as crianças não estão inclinadas a emitirem falsas declarações sem que tenham sofrido uma sugestão.

Nos anos 90, havia uma dicotomia entre profissionais que acreditavam que crianças nunca mentiam (ou falavam inverdades) e céticos que afirmavam que as crianças eram altamente sugestionáveis (Poole \& Lamb, 2009). Ainda há reflexo disso na sociedade e na academia, onde há profissionais e pesquisadores que apresentam um forte referencial de proteção da criança/ adolescente ou uma identificação prévia com a vítima, e outros que procuram ser mais céticos e objetivos, limitando-se a fatos e evidências corroborativas (Herman, 2005). Entretanto, há evidências científicas de que existem falsas acusações de abuso sexual, que tornam complexa a tarefa de avaliar (Amendola, 2009).

Ocorrências sem evidências físicas/materiais nos casos de ASI têm feito com que profissionais da saúde mental sejam cada vez mais chamados a participar e contribuir em audiências e avaliações (Dammeyer, 1998; Finnilä-Tuohimaa, Santtila, Sainio, Niemi, \& Sandnabba, 2005; Herman, 2010). Nesses casos, decidir quanto à veracidade do abuso não é tarefa simples, principalmente porque não há uma síndrome específica que diferencie vítimas de não vítimas, ou seja, algumas vítimas podem ser assintomáticas e não vítimas podem apresentar sintomas (Dammeyer, 1998; Gava, Silva, \& Dell'Aglio, 2013; Kuehnle, 1998). Por tal razão, o ASI não é tanto considerado um diagnóstico, mas muito mais um evento de vida (Kuehnle, 1998).

Muitos profissionais orientam suas práticas pela avaliação de sinais e sintomas de abuso em crianças e adolescentes. Esse evento pode de fato produzir uma grande variedade de problemas comportamentais, afetivos, psicológicos, físicos, de personalidade (Myers, 2006). Entretanto, a possibilidade de que possam ser observados sintomas não significa que eles estejam presentes: algumas vítimas podem apresentar sé- rios problemas e psicopatologias; outras podem não ter consequências tão severas; ainda outras podem não apresentar nenhuma consequência, dependendo das características da violência e da vítima (Saywitz, Mannarino, Berliner, \& Cohen, 2000). Por isso, uma avaliação de ASI não pode considerar apenas os sintomas aparentes e associá-los da seguinte forma: "presença de sintomas = ocorrência de abuso" e "ausência de sintomas = não ocorrência de abuso". Ocorre que profissionais combinam um problema específico a características específicas e deixam de considerar a possibilidade de haver características sem a ocorrência do problema (Finnilä-Tuohimaa et al., 2005). Isso faz com que o processo de tomada de decisão em situações de ASI não possa ser baseado nessas associações e deva considerar todas as evidências e influências aos quais os avaliadores estão expostos. É necessário verificar as possibilidades de esse evento ter ocorrido ou não, tendo em vista a existência de falsas acusações e falsas memórias (Amendola, 2009; Loftus, 1997; Stein, 2010).

Diante disso, as evidências psicológicas têm sido compreendidas como contribuintes para uma percepção mais acurada do caso, especialmente se a avaliação ocorre numa base científica. Porém, as mesmas evidências podem gerar conclusões diferentes em avaliações forenses (Everson \& Sandoval, 2011). Isso ocorre especialmente quando profissionais consideram apenas observações subjetivas e suas próprias experiências clínicas, subjugando o conhecimento empírico sobre o tema (Kuehnle, 1998). Geralmente, uma conclusão nessas situações diz respeito a encontrar indicadores que determinam se um evento (ASI) ocorreu ou não, diferentemente de fazer um diagnóstico (Finnilä-Tuohimaa, Santtila, Sainio, Niemi, \& Sandnabba, 2009). Entretanto, ainda que se refiram a um mesmo caso, com as mesmas evidências disponíveis, existem desacordos entre profissionais (Herman, 2005). Por isso, é necessário investigar os fatores que fazem com que, diante das mesmas evidências, profissionais avaliem e tomem decisões diferentemente.

Nas avaliações de ASI, a incerteza é um elemento frequente (Finnilä-Tuohimaa et al., 2009; 
Stokes \& Schmidt, 2012). Boas habilidades de tomada de decisão e conhecimento são fatores de expertise, mas a pesquisa não apresenta profissionais conduzindo investigações de alta qualidade (Finnilä-Tuohimaa et al., 2009). Pelo contrário, falta de adequado nível de treinamento, conhecimento e habilidades tem sido citada na literatura (Herman, 2005). Uma revisão sobre decisões em situações de ASI demonstrou que a concordância entre avaliadores é baixa, independentemente de estudos usarem entrevistas gravadas em vídeos, vinhetas, casos fictícios ou reais (Herman, 2005).

A tomada de decisão é um processo cognitivo complexo, que pode sofrer influência de muitos fatores. Em situações de ASI, a pesquisa tem demonstrado que as evidências não são os únicos fatores considerados pelos profissionais nas suas conclusões (Finnilä-Tuohimaa et al., 2009). Alguns estudos têm demonstrado que existe influência do sexo, idade e da área de conhecimento sobre a tomada de decisão: mulheres, pessoas mais jovens e assistentes sociais apresentam maiores chances de acreditarem que um caso de abuso realmente aconteceu (Jackson \& Nuttall, 1993). Mulheres usualmente apresentam um comportamento pró-criança e este efeito tem sido visto tanto em juris simulados com estudantes como em estudos mais realísticos usando membros elegíveis da comunidade e audiências reais (Bottoms, Golding, Stevenson, Wiley, \& Yozwiak, 2007). O sexo do perpetrador também tem apresentado influência sobre as tomadas de decisão em ASI (Hetherton \& Beardsall, 1998). É conhecido o fato de os homens serem os principais perpetradores deste crime, mas também existem mulheres abusadoras sexuais. Entretanto, quando um caso de ASI é apresentado envolvendo uma perpetradora mulher, geralmente é considerado um problema menos sério do que quando o suspeito é do sexo masculino (Hetherton \& Beardsall, 1998). Portanto, é fundamental considerar os vieses subjacentes às decisões profissionais que poderão afetar as vidas de vítimas e suas famílias.

Variáveis cognitivas também foram encontradas influenciando a tomada de decisão tanto em crimes como em decisões clínicas (Finnilä-
Tuohimaa et al., 2009). As crenças de profissionais sobre ASI foram investigadas num survey conduzido com 1106 profissionais da área de maus tratos (Everson \& Sandoval, 2011). Algumas pessoas acreditam que a vítima que reluta em revelar tem mais chance de não ter sofrido o abuso. Outras pessoas tendem a concluir em favor da vítima porque acreditam que, se estiverem cometendo um erro, é preferível cometê-lo em favor da vítima. Diferentemente, pessoas mais preocupadas com a objetividade e especificidade dos fatos, podem preferir correr o risco de cometer erros em favor do réu.

O processo de tomada de decisão (TD) é uma função cognitiva que torna explícitos os limites cognitivos humanos. Tendo em vista que a racionalidade não pode dar conta de todos os aspectos envolvidos em um determinado fenômeno, a cognição humana se utiliza de atalhos mentais para processar a informação num modo econômico. A TD é uma função vulnerável a vieses, erros e inconsistências (Arruabarrena \& De Paúl, 2012). As heurísticas de representatividade e disponibilidade são exemplos desse processamento simplificado, que podem levar a erros na TD (Sternberg, 2000). A heurística é um atalho mental que faz com que o processamento cognitivo utilize algumas informações e ignore outras. Na heurística da disponibilidade, aspectos mnemônicos estão envolvidos: quanto mais facilmente o sujeito pode trazer à memória determinadas informações, mais ele vai utilizá-las. As pessoas raramente têm tempo ou recursos para acessar todas as informações disponíveis antes de formar um julgamento. Ao invés disso, muitas decisões diárias são acuradamente feitas conduzindo uma busca rápida por exemplos altamente salientes ou típicos que se relacionam ao problema. Entretanto, esse atalho pode levar a erros sistemáticos em ambientes complexos. A heurística da disponibilidade é a tendência a basear julgamentos em eventos altamente salientes, memoráveis ou disponíveis. A disponibilidade pode explicar porque clínicos que se especializam em diagnósticos particulares são frequentemente criticados por diagnosticar mais frequentemente do que é esperado (Poole \& Lamb, 2009). 
Já a heurística da representatividade envolve elementos que consideramos representativos do fenômeno: quanto mais o sujeito se depara com aspectos que julga serem característicos de um determinado evento, maior a influência na credibilidade que dará a ele. É a tendência a avaliar a probabilidade de $\mathrm{A}$ estar associado a $\mathrm{B}$ em função de uma similaridade percebida entre A e B (Poole \& Lamb, 2009). Entretanto, uma similaridade superficial pode levar os profissionais a exagerarem a força da relação. Exemplo: estudos clínicos indicam altos níveis de disfunção sexual em adultos que foram vítimas de abuso. Entretanto, a disfunção sexual é explicada por várias outras condições que não somente abuso. Dessa forma, pode ser que um profissional que tenha atendido inúmeros casos de abuso sexual (disponibilidade), tenda a superestimar sua probabilidade. Outra possibilidade é a associação "sintoma-evento" (representatividade; Finnilä-Tuohimaa et al., 2005).

Outros possíveis erros no processamento da informação sobre abuso sexual podem incluir o hábito de profissionais considerarem a combinação de vários indicadores para a presença de um determinado diagnóstico, mas não se questionarem sobre se aqueles indicadores poderiam ser explicados por outra condição (reversão de probabilidades), como no exemplo: muitas crianças que foram vítimas de abuso sexual apresentam comportamento hipersexualizado, mas quantas que exibem comportamento hipersexualizado foram vítimas de abuso sexual?. Além de geralmente não reverterem o questionamento, os profissionais tendem a confirmar suas próprias hipóteses (viés de confirmação), ignorando informações que a enfraqueceriam. O questionamento do profissional, portanto, se dá na direção de aumentar as chances de achar relações entre eventos, mas limita as chances de encontrar achados contrários (Poole \& Lamb, 2009).

Outras tendências cognitivas envolvem o fato de notarmos quando eventos são pareados/ relacionados, mas falharmos em notar situações em que os eventos não são (correlação ilusória). Isto ocorre porque pares específicos vêm à mente prontamente, como por exemplo, o par "com- portamento hipersexualizado-abuso sexual", que parece ter papel significativo nesse processo. $\mathrm{O}$ ser humano também é direcionado a favorecer informações que aparecem primeiramente em um determinado problema (ancoragem e ajustamento). Entretanto, uma informação que emergiu previamente não necessariamente tem mais valor do que uma que apareceu tardiamente. As decisões sobre crianças raramente são feitas incluindo todas as informações relevantes e, geralmente, profissionais descobrem as informações pouco a pouco. Uma informação que emergiu primeiramente pode influenciar decisões futuras. Além disso, nossas decisões são influenciadas pelo modo como a informação é apresentada (problema de configuração) e pela superconfiança. Alguns profissionais apresentam superconfiança em sua habilidade de "detectar" casos de abuso sexual. Entretanto, não há uma relação entre o profissional ser autoconfiante e ter acurácia. Obter mais experiência pode tornar o profissional mais autoconfiante, mas nem experiência tampouco autoconfiança apresentam impacto sobre a acurácia (Poole \& Lamb, 2009).

Além de sexo, idade, área de conhecimento, crenças e outros aspectos cognitivos, variáveis relacionadas ao caso, como o tipo de informação apresentada, podem influenciar a TD (Peters, 2001). Vinhetas de casos envolvendo ASI foram apresentadas e avaliadas por participantes, que decidiam, a partir delas, a probabilidade de ocorrência do abuso. Os resultados indicaram que quando as vinhetas apresentavam evidências físicas, revelação detalhada e informações colaterais, as situações de ASI foram avaliadas como tendo alta probabilidade de serem reais (Peters, 2001). Além das informações sobre o caso, o comportamento da vítima também pode exercer influência sobre a TD (Goodman et al., 1992). Numa situação real de testemunho de uma vítima, ela pode demonstrar diferentes comportamentos. Num júri simulado de ASI, autores encontraram que a percepção dos jurados sobre a vítima pode modificar a TD: se a vítima está chorosa, com expressões faciais e entonação da voz demonstrando estar chateada, o júri tende a considerar uma maior probabilidade de 
ocorrência do crime (Golding, Fryman, Marsil, $\&$ Yozwiak, 2003). Isso implica em maiores vereditos de culpado ao réu quando a vítima está apresentando maior sofrimento (Regan \& Baker, 1998). Outro exemplo foi o estudo de Golding et al. (2003), que controlou variáveis como o sexo do participante e a intensidade do comportamento de choro da vítima, e observou que participantes mulheres deram mais vereditos de culpado ao réu do que os participantes homens. Além disso, a condição de "choro" da vítima, em oposição a apresentar-se calmo ou chorar histericamente, influenciou os jurados a darem mais vereditos de culpa ao réu. De acordo com Regan e Baker (1998), jurados consideram como esperado um comportamento de chorar da vítima de ASI, mas há um nível ótimo desse comportamento para que ele ganhe credibilidade (Golding et al., 2003).

A TD em situações de suspeita de abuso sexual é uma tarefa que o profissional executa e não recebe feedback sistemático (Poole \& Lamb, 2009). Isso significa que o profissional não poderá receber uma resposta, ao final de seu trabalho, sobre se ele chegou ou não a uma decisão verdadeira. Ao trabalhar sem ter conhecimento sobre estar tomando ou não decisões corretas, os profissionais acabam desenvolvendo um determinado procedimento de trabalho, baseados em teorias próprias que incluem estratégias cognitivas. Tendo em vista os diferentes fatores que podem afetar a tomada de decisão em situações envolvendo abuso sexual, o objetivo deste estudo foi investigar como profissionais de Psicologia tomam decisões sobre um possível e hipotético caso de suspeita de abuso sexual contra uma criança e verificar as relações entre a tomada de decisão e variáveis biosociodemográficas, profissionais e cognitivas.

\section{Método}

\section{Delineamento}

Foi realizado um survey online. O site que abrigou os instrumentos de coleta de dados permaneceu online por oito meses, entre setembro de 2011 e maio de 2012, tendo sido divulgado eletronicamente por todo o país.

\section{Participantes}

Responderam de forma completa aos instrumentos 95 psicólogos ( $86,3 \%$ do sexo feminino) das cinco regiões do Brasil (54,7\% da região sul; $22,1 \%$ sudeste; $10,5 \%$ norte; $7,4 \%$ nordeste e $5,3 \%$ centro-oeste), com idade entre 23 e 62 anos $(M=36,6 ; D P=9,7)$ e tempo de formação entre 10 meses e 34 anos $(M=11,2 ; D P=8,8)$. Os participantes indicaram que atuavam em situações de suspeita de abuso sexual contra crianças e adolescentes entre menos de um ano e 34 anos $(M=10,7 ; D P=8,8)$, com uma média de casos avaliados de $34,1(D P=80,2)$.

\section{Instrumentos}

Foram utilizados os seguintes instrumentos: Questionário de Dados Biossociodemográficos: para investigar dados relativos ao sexo, idade, número de casos avaliados; tempo de formação; tempo de atuação em situações de ASI e familiaridade auto avaliada com a literatura.

Cenário 7 (Herman \& Freitas, 2010): é um caso hipotético desenvolvido nos Estados Unidos e traduzido para a língua portuguesa para esse estudo. A tradução para a língua portuguesa foi realizada em três etapas: (a) dois juízes bilíngues traduziram o instrumento; (b) um grupo de juízes pesquisadores e experientes em leitura em outros idiomas verificou a adequação, sugerindo algumas alterações; (c) uma juíza bilíngue moradora de país de língua inglesa e pesquisadora da área de ciências humanas verificou e aprovou a tradução. Este "cenário" apresenta um caso de suspeita de ASI e convida o participante a optar por uma decisão: ou ele "fundamenta" (quando entende que há subsídios suficientes para afirmar que o abuso ocorreu) o caso ou "não fundamenta" (quando entende que não há subsídios suficientes). Além dessa questão dicotômica, os participantes avaliavam a probabilidade de o abuso ter ocorrido numa escala de 0 a $100 \%$ de certeza. Importante ressaltar que o caso apresenta dados ambíguos, é fictício e não tem uma resposta "correta", previamente determinada. O caso apresenta tanto elementos que induzem o leitor a acreditar na ocorrência do abuso (por exemplo, quando a personagem "Laura" desenha órgãos 
sexuais masculinos em sala de aula e diz que seu pai é "mau") como também elementos de dúvida (por exemplo, quando Laura parece confusa na primeira entrevista e nega qualquer contato sexual).

Escala de Tomada de Decisão em Situações de Abuso Sexual Infantil (ETD-ASI): foi elaborada pela equipe de pesquisa, a partir da revisão da literatura para avaliar a influência do pensamento representativo na tomada de decisão em casos de suspeita de abuso sexual contra crianças e adolescentes. Este instrumento inclui 15 itens que abordam aspectos representativos dos casos de abuso sexual infantil e utiliza uma escala Likert de cinco pontos, indo do "Discordo totalmente" ao "Concordo totalmente". Os itens foram baseados na literatura especializada e a escala teve seu conteúdo avaliado por três diferentes juízes com reconhecido conhecimento na área do abuso sexual contra crianças e adolescentes, com mestrado e/ou doutorado. A soma dos itens gera um escore total que indica a influência do pensamento representativo na avaliação das situações de ASI. Uma análise da confiabilidade foi conduzida e indicou um Alpha de Cronbach de 0,89 . Numa análise por item, verificou-se que a exclusão de itens não implicaria no aumento do Alpha, optando-se por permanecer com todos os 15 itens.

\section{Procedimentos e Considerações Éticas}

$\mathrm{O}$ projeto de pesquisa foi aprovado pelo $\mathrm{Co}-$ mitê de Ética em Pesquisa do Instituto de Psicologia da Universidade Federal do Rio Grande do Sul - UFRGS, sob o protocolo 20698. Os participantes foram convidados por via eletrônica, num método conhecido como "bola de neve" ou snow ball. Por e-mails, os profissionais eram convidados a participar do estudo, caso se enquadrassem nos critérios de inclusão (ser psicólogo, ter um registro ativo em seu respectivo Conselho Regional e ter atuado na avaliação de pelo menos dois casos de suspeita de abuso sexual de crianças e adolescentes). Os participantes poderiam atuar em diferentes setores, seja público ou privado. Seguindo as orientações para pesquisa com seres humanos no Brasil, um Termo de Consentimento Livre e Esclarecido - TCLE era a primeira parte do questionário online. Sem a concordância do participante, ele não poderia seguir adiante no instrumento.

\section{Análise de Dados}

Os dados obtidos foram analisados utilizando-se o Statistical Package for Social Sciences SPSS 20. Foram analisados de forma descritiva os dados sociodemográficos (variáveis independentes) e os resultados do Cenário 7 e do ETD-ASI (variáveis dependentes). Foram realizados testes de correlações entre as variáveis quantitativas investigadas, assim como teste $t$ para verificar diferenças por sexo.

\section{Resultados}

Primeiramente, o instrumento Cenário 7 indicou dois principais resultados descritivos: 43 participantes $(45,3 \%)$ não fundamentaram o caso enquanto $52(54,7 \%)$ fundamentaram, embora essa diferença não seja significativa $\left(X^{2}=\right.$ $0,85 ; g l=1 ; p>0,35)$. Quanto à probabilidade de que o caso tenha ocorrido, a distribuição dos resultados é apresentada na Tabela 1.

\section{Tabela 1}

Distribuição da Probabilidade de Ocorrência do Caso

\begin{tabular}{ccc}
\hline Probabilidade & $f$ & $\%$ \\
\hline 0 & 2 & 2,1 \\
10 & 2 & 2,1 \\
20 & 2 & 2,1 \\
30 & 4 & 4,2 \\
40 & 4 & 4,2 \\
50 & 53 & 55,8 \\
60 & 4 & 4,2 \\
65 & 1 & 1,1 \\
70 & 4 & 4,2 \\
80 & 4 & 4,2 \\
90 & 8 & 8,4 \\
95 & 1 & 1,1 \\
100 & 6 & 6,3 \\
\hline Total & 95 & 100 \\
\hline
\end{tabular}

Os resultados descritivos do Cenário 7 indicam claramente uma tendência central dos participantes, indicando que num caso ambíguo 
como o descrito, os psicólogos preferem optar por indicar uma probabilidade média de ter ocorrido o caso. Observa-se, que quando forçados a responderem dicotomicamente sobre a ocorrência real do abuso, os participantes apresentam opiniões diferentes, como observado na distribuição dos casos em que fundamentaram ou não. Análises correlacionais foram conduzidas e indicaram que a probabilidade de ocorrência do caso não apresentou correlação significativa com as variáveis biosociodemográficas e profissionais avaliadas (idade, tempo de formação, tempo de atuação em situações de ASI, número de casos avaliados e familiaridade auto avaliada com a literatura). Para a variável sexo, um Teste $t$ foi conduzido e não indicou diferenças entre homens $(M=58,46 ; D P=20,35)$ e mulheres $(M$
$=55,49 ; D P=22,02)$ quanto à probabilidade indicada de ocorrência do caso.

Por sua vez, os resultados da ETD-ASI variaram entre 18 e $73(M=49,7 ; D P=11,3)$, considerando que a variabilidade possível do instrumento era de 15 a 75 . As médias por item podem ser verificadas na Tabela 2. Ainda nesta tabela são apresentadas análises correlacionais entre os itens da ETD-ASI e a probabilidade de ocorrência do caso obtida no Cenário 7. A correlação entre o escore total da ETD-ASI e a probabilidade de ocorrência do Cenário $7(r=0,32 ; p<0,01)$ indicou que a credibilidade que o profissional deu ao caso relacionou-se com a representatividade, demonstrando que itens representativos de situações de abuso sexual estão relacionados à tomada de decisão nesse caso hipotético.

\section{Tabela 2}

Médias nos Itens da ETD-ASI e Correlações com a Probabilidade de Ocorrência do Caso

\begin{tabular}{cccc}
\hline Itens da ETD-ASI & $M$ & $D P$ & $r$ \\
\hline Idade da vítima & 2,77 & 1,41 & $0,31(p<0,01)^{*}$ \\
Gênero da vítima & 2,42 & 1,32 & $0,22(p<0,03)^{*}$ \\
Relato da vítima & 4,05 & 0,99 & $0,21(p<0,05)^{*}$ \\
Características da criança/adolescente & 3,79 & 1,10 & $0,24(p<0,03)^{*}$ \\
Idade do perpetrador & 3,01 & 1,34 & $0,33(p<0,01)^{*}$ \\
Gênero do perpetrador & 2,95 & 1,34 & $0,16(p>0,10)$ \\
Antecedente criminal & 2,92 & 1,31 & $0,22(p<0,04)^{*}$ \\
Características do perpetrador & 3,63 & 1,21 & $0,10(p>0,30)$ \\
Representatividade & 3,72 & 1,06 & $0,07(p>0,50)$ \\
Separação dos pais & 2,73 & 1,28 & $0,80(p>0,40)$ \\
Sintomas & 3,73 & 1,17 & $0,22(p<0,04)^{*}$ \\
Comportamento hipersexualizado & 3,97 & 0,97 & $0,24(p<0,02)^{*}$ \\
Família reconstituída & 2,34 & 1,05 & $0,16(p>0,10)$ \\
Características maternas & 3,57 & 1,02 & $0,22(p<0,04)^{*}$
\end{tabular}

$* p<0,05$.

A análise dos resultados pode indicar o quanto cada aspecto dos casos impacta na TD dos profissionais. Observando as médias de cada item, percebe-se que os itens mais impactantes na decisão foram o relato da vítima, as características da criança/adolescente, a representatividade da dinâmica do caso relatado, os sintomas apresentados pela suposta vítima, o comportamento hipersexualizado e a coerência da narrativa. 


\section{Discussão}

Este estudo avaliou a tomada de decisão em situações de ASI por profissionais da Psicologia e a relação com variáveis biosociodemográficas, profissionais e cognitivas. É relevante destacar o fato de o mesmo caso ter sido apresentado a todos os participantes e estes, forçados a uma decisão sobre o abuso ter ou não ocorrido, tomaram decisões diferentes, embora a maioria tenha se posicionado de forma neutra. Pode-se considerar que os psicólogos se dividiram na metade: uma parte "fundamentou" o abuso e outra metade "não fundamentou", confirmando resultados outrora destacados na literatura sobre a não concordância entre profissionais (Herman, 2005). Além disso, a tendência central apresentada pelos participantes demonstra a incerteza e a ambiguidade de caso fictício utilizado, que é semelhante à ambiguidade real e frequentemente enfrentada pelos profissionais, justificando a presença de uma inevitável incerteza para a tomada de decisão em muitos casos na vida real (Finnilä-Tuohimaa et al., 2009; Stokes \& Schmidt, 2012). Novos estudos devem testar como os participantes agem em casos ambíguos para eliminar suas incertezas (por exemplo se buscam novas evidências) e como eles tomam decisão em casos prototipicamente fundamentáveis ou não, ou, ainda mais interessante, em casos distribuídos em um continuum de ambiguidade.

Diferentemente do apresentado na literatura, as variáveis biosociodemográficas e profissionais avaliadas não apresentaram relação com a decisão tomada no caso fictício. Idade e sexo, por exemplo, não influenciaram a TD nesta amostra, indicando que pouco importa o psicólogo ser mais velho ou mais jovem, homem ou mulher. Esses dados contrariam os achados de Bottoms e colaboradores (2007), Finnilä-Tuohimaa et al. (2005), Hetherton e Beardsall (1998) e Jackson e Nuttall (1993). Além disso, a experiência, avaliada a partir do número de casos, tempo de formação e tempo de atuação, também não apresentou relação com a TD. No entanto, neste estudo, a hipótese da heurística da disponibilidade (Poole \& Lamb, 2009) não pode ser avaliada integralmente, requerendo novos estudos. Os resultados encontrados, portanto, não permitem afirmar que um profissional, por atender muitos casos de ASI ou por ter muito tempo de formação e de atuação, vai considerar mais provável um próximo caso ser real, do que seu colega menos experiente, diferentemente do resultado encontrado por Finnilä-Tuohimaa et al. (2005). Outra variável que não se mostrou associada à probabilidade de ocorrência do caso foi a familiaridade auto avaliada com a literatura sobre ASI, que pode ser compreendida como a autoconfiança do profissional sobre o seu conhecimento (Poole \& Lamb, 2009).

Entretanto, as características representativas dos casos de abuso sexual, ilustradas pelas questões da ETD-ASI, tiveram relações significativas com a probabilidade de ocorrência do abuso evidenciada no Cenário 7. Assim, os participantes consideram que determinados aspectos estão relacionados à ocorrência do abuso, tais como o relato da vítima, as características da criança/ adolescente, a representatividade da dinâmica do caso relatado, os sintomas apresentados pela suposta vítima, o comportamento hipersexualizado e a coerência da narrativa, fortalecendo a hipótese da influência da heurística da representatividade na tomada de decisão em situações de ASI (Poole \& Lamb, 2009).

As características da criança/adolescente, os sintomas apresentados e o comportamento hipersexualizado podem ser teoricamente compreendidos como representativos do impacto do abuso sexual sobre a vítima. Diversos estudos identificaram as possíveis consequências do abuso sexual e, tradicionalmente, os achados clínicos costumam pesar muito na TD em ASI (Myers, 1993). Os resultados de Paolucci, Genius e Violato (2001), entretanto, suportaram a hipótese de um modelo multifacetado do trauma em oposição a uma síndrome específica do ASI. Esse modelo indica que muitas combinações de diferentes sintomas podem ser efeito da ocorrência de um ASI (Paolucci et al., 2001), que pode haver vitimização sem sintomatologia ou ainda que muitos sintomas podem ser indicativos de outros problemas que não ASI (Finnilä-Tuohimaa et al., 2005, 2009). 
Especificamente, um possível efeito do ASI que chama a atenção dos avaliadores e tomadores de decisão é o comportamento hipersexualizado. De fato, as demonstrações deste comportamento devem aumentar a preocupação em relação à possibilidade de ASI (Dammeyer, 1998). Entretanto, comportamento hipersexualizado pode ser definido diferentemente entre culturas e também pode ser produto de uma exposição acidental à pornografia ou conteúdo sexual impróprio para a idade, diferente de uma vitimização direta. Por isso, ele deve ser observado atentamente e não pode ser compreendido como uma evidência comprobatória de que o abuso ocorreu, mas sim, de que possa ter ocorrido, mas que merece maior investigação.

O ASI é, em grande parte das vezes caracterizado por uma dinâmica específica. A presença de ameaças, por exemplo, é comumente relatada pelas vítimas. Barganhas, segredos e uma evolução gradual de um contato mais sutil a mais agressivo também são descritos na literatura como característicos. No presente estudo, um relato que compreenda uma história que inclua elementos já conhecidos influencia a TD, como indicado na seção de resultados. Segundo Dammeyer (1998), o relato da criança/adolescente e os exames médicos são as melhores fontes de informação em casos de ASI. É consenso entre profissionais que se deve confiar muito no relato da criança. Entretanto, sabe-se que uma criança pode produzir um relato claro de um evento fictício após ser treinada para isso, como em alguns casos de separação e disputa de guarda, ou serem sugestionadas por pais ou profissionais com perguntas inadequadas (Bernet, 2010).

Dessa forma, é importante esclarecer que o relato da vítima deve ter mais peso quando fora dessas situações de disputa de guarda e sugestionabilidade. $\mathrm{O}$ relato da vítima ganha mais peso quando ele é coerente. Para Pennington e Hastie $(1988,1992)$, quando o corpo de evidências é grande e complexo, o processo de tomada de decisão é baseado em explicação. Assim, em decisões baseadas em explicação, os tomadores de decisão constroem um modelo causal para explicar os fatos disponíveis. O "Modelo da História em Decisões Judiciais" prevê que as decisões são tomadas a partir de uma narrativa em que relações entre eventos são centrais. A organização da história facilita a compreensão das evidências e possibilita a tomada de decisão. Para as autoras, durante uma situação de julgamento em um tribunal, os tomadores de decisão estão envolvidos em um processamento mental ativo de construção de uma representação mental coerente do caso. As informações são apresentadas pouco a pouco, a partir de perguntas e respostas realizadas na própria audiência e evidências coletadas previamente. Muitas informações são apresentadas de forma "desconectada". De acordo com este modelo, a representação mental do problema é construída combinando informações do caso específico, conhecimento de situações similares e uma expectativa geral sobre o que faz uma história ser completa. Dessa forma, se as informações são coerentes e aparecem em ordem cronológica, a compreensão fica facilitada e direciona a TD. Um jurado vai construir uma história do que ocorreu e, tendo alta coerência, essa história é mais facilmente aceita como verdadeira (Klettke, Graesser, \& Powel, 2010; Pennington \& Hastie, 1988, 1992).

\section{Considerações Finais}

Neste estudo, observou-se a complexidade da TD em ASI, com psicólogos tendo opiniões diferentes sobre o caso, mesmo tendo acesso às mesmas informações. As influências sobre essas decisões não foram de cunho biosociodemográfico (sexo ou idade), tampouco profissionais (tempo de graduação, de atuação, número de casos ou familiaridade com a literatura). Entretanto, o conhecimento sobre aspectos representativos de ASI parece exercer influência significativa sobre a TD no sentido de os profissionais terem mais probabilidade de fundamentar o caso quanto mais este for similar aos casos descritos na literatura. Ainda que seja necessário o conhecimento sobre a dinâmica e sinais e sintomas, os profissionais não devem considerar isoladamente nenhum aspecto como confirmação da ocorrência do abuso. É desejável também que os questionamentos/entrevistas não sejam direcionados de modo a excluir outras possibilidades de 
desfecho para o caso. A consciência de vieses pessoais é fundamental na condução de avaliações em ASI (Dammeyer, 1998).

Decisões sobre estes casos são complexas e devem ser feitas com muito cuidado, uma vez que frequentemente implicam em grande impacto na vida das crianças/adolescentes vítimas e suas famílias. Por essa complexidade, dificuldade e por seus efeitos, a TD pode se beneficiar de check lists que estruturem a TD, como tem sido recentemente realizado na Alemanha em casos de maus-tratos contra crianças (Kwaadsteniet, Bartelink, Witteman, Berge, \& Yperen, 2013). O objetivo é tornar a TD menos subjetiva, mais transparente, sistemática, uniforme e eficiente. Métodos mais objetivos de TD em situações de maus tratos contra crianças também têm sido investigados na Austrália (Victorian Risk Framework - VRF - Armitage, Boffa, \& Parker, 1999), Estados Unidos (Structured Decision Making - SDM - Children's Research Center, 1999) e Espanha (Arruabarrena \& De Paul, 2012). Em virtude da complexidade deste processo, é fundamental que os profissionais tenham cuidado ao elaborar seus documentos, considerando que as avaliações psicológicas podem indicar probabilidades de ocorrência da situação de violência sexual, mas devem evitar oferecer certezas não fundamentadas. É importante considerar, ainda, os diferentes papéis profissionais, entendendo que as decisões sobre ocorrência e responsabilização cabem aos operadores do Direito, enquanto que ao psicólogo cabe realizar entrevistas, avaliações e atuar no sentido da proteção e garantia aos direitos de todos os envolvidos (Pelisoli \& Dell'Aglio, 2014).

As limitações deste estudo incluem o número de participantes e a utilização de um único caso fictício e ambíguo para verificar a tomada de decisão. Dessa forma, novos estudos mais abrangentes que envolvam diferentes casos (reais e fictícios), utilizando vinhetas, juris simulados e outros recursos podem trazer contribuições relevantes à literatura. É importante também considerar a necessidade de investigar os erros cometidos por profissionais que avaliam estes casos e maneiras de evitá-los, preservan- do vítimas e familiares. Tomar decisões em circunstâncias de ASI é rotina para profissionais de diferentes áreas, não somente da Psicologia, e implica em consequências determinantes na vida das pessoas envolvidas nessas situações. Investigar a influência de fatores como sexo, idade, experiência profissional, crenças, entre outros significa conhecer os processos que envolvem a TD em profissionais e significa, consequentemente, a qualificação da atuação dos profissionais que trabalham com esse problema.

\section{Referências}

Amendola, M. F. (2009). Analisando e (des)construindo conceitos: Pensando as falsas denúncias de abuso sexual. Estudos e Pesquisas em Psicologia, 9(1), 199-218.

Armitage, E., Boffa, J., \& Parker, B. (1999). Victorian risk framework: A guided professional judgment approach to risk assessment in child protection. Victoria, Australia: Department of Human Services.

Arruabarrena, I., \& De Paúl, J. (2012). Improving accuracy and consistency in child maltreatment severity assessment in child protection services in Spain: New set of criteria to help caseworkers in substantiation decisions. Children and Youth Services Review, 34, 666-674. doi:10.1016/j. childyouth.2011.12.011

Berliner, L., \& Conte, J. R. (1993). Sexual abuse evaluations: Conceptual and empirical obstacles. Child Abuse \& Neglect, 17, 111-125. doi:10.1016/0145-2134(93)90012-T

Bernet, W. (2010). Falsas denúncias e o diagnóstico diferencial das alegações de abuso sexual. Psicologia Revista São Paulo, 19(2), 263-288.

Bottoms, B. L., Golding, J. M., Stevenson, M. C., Wiley, T. R. A., \& Yozwiak, J. A. (2007). A review of factors affecting jurors' decisions in child sexual abuse cases. In M. Toglia, J. D. Read, D. F. Ross, \& C. L. Lindsay (Eds.), Handbook of eyewitness psychology: Vol. 1. Memory for events (pp. 509-543). Mahwah, NJ: Lawrence Erlbaum.

Children's Research Center. (1999). The improvement of child protective services with structured decision making: The CRC model. Madison, WI: Children's Research Center. 
Dammeyer, M. D. (1998). The assessment of child sexual abuse allegations: Using research to guide clinical decision making. Behavioral Sciences and the Law, 16, 21-34. doi:10.1002/ (SICI)1099-0798(199824)16:1\%3C21::AIDBSL291\%3E3.0.CO;2-K

Everson, M. D., \& Sandoval, J. M. (2011). Forensic child sexual abuse evaluations: Assessing subjectivity and bias in professional judgements. Child Abuse and Neglect, 35(4), 287-298. doi:10.1016/j.chiabu.2011.01.001

Finnilä-Tuohimaa, K., Santtila, P., Sainio, M., Niemi, P., \& Sandnabba, K. (2005). Connections between experience, beliefs, scientific knowledge and self-evaluated expertise among investigators of child sexual abuse in Finland. Scandinavian Journal of Psychology, 46, 1-10. doi:10.1111/j.1467-9450.2005.00429.x

Finnilä-Tuohimaa, K., Santtila, P., Sainio, M., Niemi, P., \& Sandnabba, K. (2009). Expert judgment in cases of alleged child sexual abuse: Clinicians'sensitivity to suggestive influences, pre-existing beliefs and base rates estimates. Scandinavian Journal of Psychology, 50, 129 142. doi:10.1111/j.1467-9450.2008.00687.x

Gava, L. L., Silva, D. G., \& Dell'Aglio, D. D. (2013). Sintomas e quadros psicopatológicos identificados nas perícias em situações de abuso sexual infanto-juvenil. Psico, 44(2), 235-244.

Golding, J. M., Fryman, H. M., Marsil, D. F., \& Yozwiak, J. A. (2003). Big girls don't cry: The effect of child witness demeanor on juror decisions in a child sexual abuse trial. Child Abuse \& Neglect, 27(11), 1311-1321. doi:10.1016/j. chiabu.2003.03.001

Goodman, G. S., Taub, E. P., Jones, D. P. H., England, P., Port, L. K., Rudy, L., \& Prado, L. (1992). Testifying in criminal court: Emotional effects on child sexual assault victims. Monographs of the Society for Research in Child Development, 57(5, Serial No. 229), 1-142.

Herman, S. (2005). Improving decision making in forensic child sexual abuse evaluations. Law and Human Behavior, 29(1), 87-120. doi:10.1007/ s10979-005-1400-8

Herman, S. (2010). The role of corroborative evidence in child sexual abuse evaluations. Journal of Investigative Psychology and Offender Profiling, 7, 189-212. doi:10.1002/jip.122
Herman, S., \& Freitas, T. R. (2010). Error rates in forensic child sexual abuse evaluations. Psychological Injury and Law, 3, 133-157. doi:10.1007/ s12207-010-9073-0

Hetherton, J., \& Beardsall, L. (1998). Decisions and attitudes concerning child sexual abuse: Does the gender of the perpetrator make a difference to child protection professionals? Child Abuse \& Neglect, 22(12), 1265-1283. doi:10.1016/s01452134(98)00101-x

Jackson, H., \& Nuttall, R. (1993). Clinician responses to sexual abuse allegations. Child Abuse \& Neglect, 17(1), 127-143. doi:10.1016/01452134(93)90013-U

Kwaadsteniet, L., Bartelink, C., Witteman, C., Berge, I., \& Yperen, T. (2013). Improved decision making about suspected child maltreatment: Results of structuring the decision process. Children and Youth Services Review, 35, 347-352. doi:10.1016/j.childyouth.2012.11.015

Klettke, B., Graesser, A. C., \& Powel, M. B. (2010). Expert testimony in child sexual abuse cases: The effects of evidence, coherence and credentials on juror decision making. Applied Cognitive Psychology, 24, 481-494. doi:10.1002/acp.1565

Kuehnle, K. (1998). Child sexual abuse evaluations: The scientist-practitioner model. $B e-$ haviour Science Law, 16, 5-20. doi:10.1002/ (SICI) 1099-0798(199824)16:1\%3C5::AIDBSL290\%3E3.0.CO;2-3

Loftus, E. (1997). Creating false memories. Scientific American, 277(3), 70-75. doi:10.1038/scientificamerican0997-70

Myers, J. E. B. (1993). Expert testimony regarding child sexual abuse. Child Abuse \& Neglect, 17, 175-185. doi:10.1016/0145-2134(93)90017-Y

Myers, J. E. B. (2006). Child protection in America: Past, present and future. New York: Oxford University Press.

Paolucci, E. O., Genuis, M. L., \& Violato, C. (2001). A meta-analysis of the published research on the effects of child sexual abuse. The Journal of Psychology, 135(1), 17-36. doi:10.1080/00223980109603677

Pelisoli, C., \& Dell'Aglio, D. D. (2014). As contribuições da Psicologia para o sistema de justiça. Psicologia: Ciência e Profissão, 34(4), 916-930.

Pennington, N., \& Hastie, R. (1988). Explanationbased decision making: Effects of memory 
structure on judgment. Journal of Experimental Psychology, 14(3), 521-533. doi:10.1037//02787393.14.3.521

Pennington, N., \& Hastie, R. (1992). Explaining the evidence: Tests of the story model for juror decision making. Journal of Personality and Social Psychology, 62(2), 189-206. doi:10.1037//00223514.62.2.189

Peters, D. F. (2001). Examining child sexual abuse evaluations: The types of information affecting expert judgment. Child Abuse \& Neglect, 25, $149-178$

Poole, D. A., \& Lamb, M. E. (2009). Investigative interviews of children: A guide for helping professionals. Washington, DC: American Psychological Association.

Regan, P. C., \& Baker, S. J. (1998). The impact of child witness demeanor on perceived credibility and trial outcome in sexual abuse cases. Journal of Family Violence, 13(2), 187-195. doi:10.1023/A:1022845724226
Saywitz, K. J., Mannarino, A. P., Berliner, L., \& Cohen, J. A. (2000). Treatment of sexually abused children and adolescents. American Psychologist, 55(9), 1040-1049. doi:10.1037/0003066x.55.9.1040

Stein, L. M. (2010). Falsas memórias: Fundamentos cientificos e suas aplicações clínicas e jurídicas. Porto Alegre, RS: Artmed.

Sternberg, R. J. (2000). Tomada de decisão e raciocínio. In R. J. Sternberg (Ed.), Psicologia Cognitiva (pp. 339-370). Porto Alegre, RS: Artmed.

Stokes, J., \& Schmidt, G. (2012). Child protection decision making: A factorial analysis using case vignetes. Social Work, 57(1), 83-90. doi:10.1093/ sw/swr007

Recebido: $22 / 04 / 2015$

$1^{a}$ revisão: $22 / 07 / 2015$

Aceite final: 11/08/2015 